\title{
Laying the Groundwork for Assisted Rehabilitation
}

\author{
Rita Pereira Tiago Guerreiro Hugo Nicolau Daniel Gonçalves Joaquim Jorge \\ Technical University of Lisbon / INESC-ID \\ R. Alves Redol, 9, 1000-029 \\ Lisbon, Portugal \\ rita.t.c.pereira@ist.utl.pt, \{tjvg, hman\}@ vimmi.inesc-id.pt, \{daniel.goncalves, jaj\}@inesc-id.pt
}

\begin{abstract}
Motivation - To provide to physical therapists a monitoring system with effective and accurate patient monitoring and evolution analysis.
\end{abstract}

Research approach - We analyzed therapy sessions with tetraplegic patients to better understand the rehabilitation process and highlight the major requirements for a technology-enhanced tool. We developed a prototype able to automate and improve the current monitoring and follow-up processes.

Findings/Design - Preliminary results indicate that computational movement analysis and comparison can improve the quality of a rehabilitation session and overall patient evolution analysis.

Research limitations/Implications - Analysis and studies have been performed in a rehabilitation centre with a limited set of therapists (3) and patients (7).

Originality/Value - The research herein contributes with a requirement analysis for a computer-assisted rehabilitation platform. We present a tracking-based system instantiating these requirements and outline its values after a preliminary informal validation.

Take away message - The capture and virtual playback of motion in physical therapy sessions increases therapist awareness of patient condition and evolution thus improving the rehabilitation process.

\section{Keywords}

Physical Rehabilitation, Tetraplegic, Therapists, Movement Analysis, Computer-Assisted Rehabilitation.

\section{INTRODUCTION}

Recent decades brought advances in technology able to improve the life quality of people with disabilities. However, the practical developments are still scarce to what tetraplegic patients and their rehabilitation is concerned. A physiotherapist has the role to observe, interpret and act to optimize the patient's response abilities. These therapeutic processes are lengthy and require great dedication and motivation from both the therapist and the patient. Also, they consist in carrying out repetitive movement patterns across sessions, giving relevance to a suitable observation, analysis and accompaniment process.
This research aims to develop the tools at the therapists reach and hence improve the overall rehabilitation process. To this end, we studied the daily routines, session and analysis procedures at a rehabilitation centre, and contribute with a set of limitations and requirements for a technologic-enhanced rehabilitation solution for therapists. Our aim is to provide to physical therapists a computer system, with efficient and accurate mechanisms, for monitoring their patients. These mechanisms include: saving all the movements that the patient performed for further evaluation and visualization, which is likely to improve the exchange of data between therapists; keep the information for posterior analysis; have a precise measure of the patients and their evolution; and being able to compare two movements performed in different sessions.

In this paper, we present the major outcomes from the task analysis performed with the main stakeholders in therapy and rehabilitation centre. Further, building on the requirements retrieved from the aforementioned analysis, we present a work-in-progress virtual rehabilitation platform and the results from its preliminary informal evaluation.

\section{A GLIMPSE ON CURRENT PROCEDURES}

To better understand the rehabilitation process we carried out a 10 day study in a rehabilitation centre with the main stakeholders, i.e. therapists and patients (Figure 1). While we believe our findings to be valuable for the general population, we focused our studies on tetraplegic patients as the duration of the therapy may extend for several months and, in most cases, years. This time span demands a good analysis, storage and

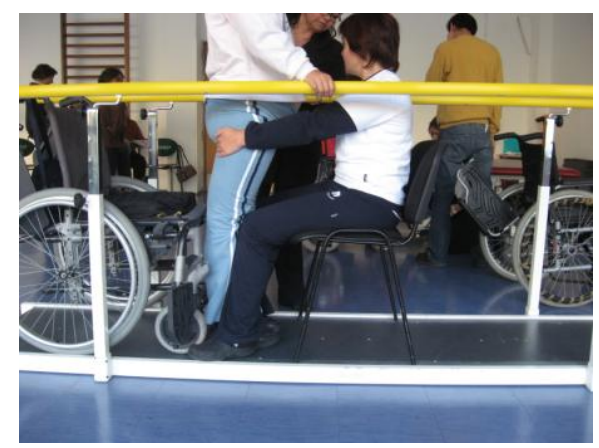

Figure 1 - Traditional Rehabilitation (Task Analysis) 
follow-up process. We performed the task analysis with 3 physiotherapists and 7 tetraplegic patients. It consisted on interviews and questionnaires to all the intervenient parts, and a thorough observation of therapy exercises. The interviews were performed after the sessions to understand the goals, limitations and difficulties of each method. We highlight the following conclusions:

- Most exercises are performed physically close to the therapist. During its execution, the therapist is unable to take notes or even have a full view of the performed motions or strengths (Figure 1);

- Some exercises are performed locally (e.g., moving an arm) but, to be performed correctly, depend on a set of restrictions (e.g., maintaining the trunk steady). Performing the exercises repeatedly wrong may have a hazardous effect on the patient's rehabilitation. It is hard for the therapist to have a complete view when engaged with the exercise;

- Even the movements observed by the therapist are registered with an approximate value. This value may vary from a therapist to another. Further, considering the longevity of the process it is impossible to guarantee coherence across evaluations thus damaging the record of the user's evolution;

- The patients have no visual feedback on their movement or distance to an accurate movement.

From the analysis, we consider that a computer platform supported with an accurate tracking system is a valuable addition to the current rehabilitation procedures. In the proposed system, it is important to highlight both the patients and the physiotherapists as the target populations. For therapists, this system will bring benefits such as information sharing, movement patterns, analysis and cross-movement analysis; for patients as it may increase the motivation to achieve the proper movement, as they may have feedback on their status.

\section{COMPUTER-ASSISTED VIRTUAL REHABILITATION}

The analysis performed to the current rehabilitation procedures pointed out several flaws and limitations concerning the immediate feedback, and afterwards when a thorough analysis or comparison is required. To overcome the aforementioned issues, we have developed a computer-assisted virtual rehabilitation platform considering the following requirements:

- Data persistence: All data must be persistent and coherent, so it can be visualized afterwards and shared by physiotherapists.

- Motion Capture: It should be possible to record the motion performed for posterior data analysis and reproduction;

- Accuracy: The platform should enable accurate and precise notion of a particular motion, e.g., reach of a patient's hand;
- Movement Reproduction: It should be possible to reproduce the motion at any time for analysis and evaluation of the movement;

- Movement Comparison: It should be possible to reproduce two movements overlapped, so they can be compared, e.g., to evaluate evolution;

- Automatic Information Extraction: It should be able to enrich the view and ease the analysis with information, e.g., automatically present the distance between two points in a particular movement comparison;

- Easy setup: The therapists should be able to prepare an exercise with little effort and no particular technical computer-wise expertise.

The following sections present how we have tackled these requirements.

\section{Tracking the Patients' Movements}

To accomplish the goals and assure that the requirements are fulfilled, our approach uses a virtual marker-based tracking system, where tracking of the movement is achieved through light-reflecting markers placed on the human body (Zhou, 2004) (Figure 2).

The choice of such a system, motion capture, is mostly
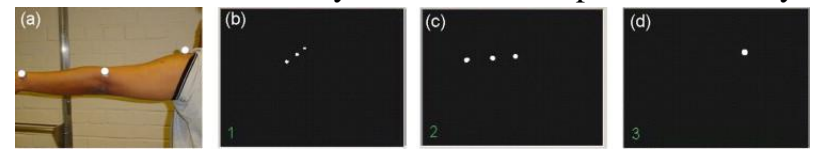

Figure 2 - Example system Virtual Marker Based

due to its precision. Moreover, it allows the monitoring of several different points at once, some of them directly related with each other (two points in the arm as in Figure 2), but others with indirect relations (trunk and arms). The latter enable the therapists to analyze posture or any erroneous movement produced.

It is relevant to notice that although we maintain an internal notion of skeleton and where the markers are placed in the human body, we do not use rigid bodies. In other words, the markers are isolated points in space, enabling the therapists to freely select the positions to follow.

To identify and be constantly aware of a particular point and its relation to others, even when it is crossed by them, we use Kalman Filters (Welch, 1995). The Kalman filter assumes that the probability density function at each instant of time follows a Gaussian distribution. This filter allows the estimation of state of a system, to minimize the mean square error, making this an optimal solution to tracking of a movement, but only if certain restrictions are met: if the noise has a Gaussian distribution known parameters and if the state transition represented by the system's model is linear (that is the case) (Welch, 1995) (Arulampalam, 2002).

The Kalman filter has two distinct phases: Predict and Update. The predict phase uses the state estimate from the previous step to produce an estimate of the state at 


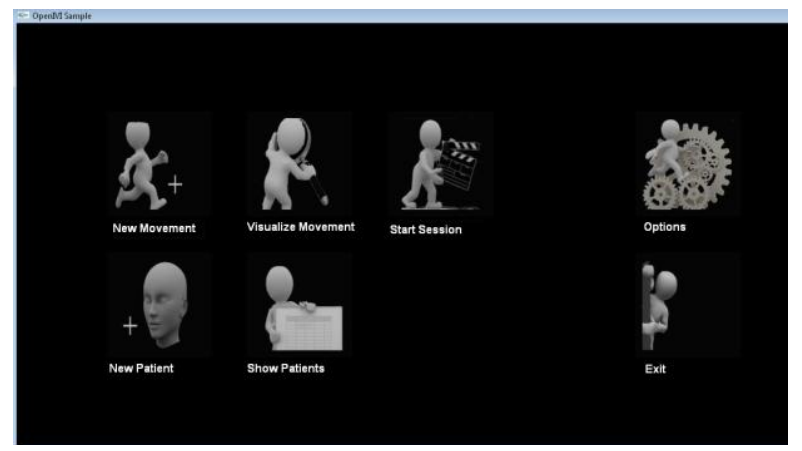

Figure 3 - Application Main Menu

the current step. In the update phase, the current "a priori" prediction is combined with current observation information to refine the state estimate.

However, this value is not enough to identify all the received points. To achieve this goal, we used the Mahalanobis distance (Pinho, 2005) and determined which of the measured points is the closest to the calculated prediction.

This process is repeated every step, with the new estimate positions and its covariance informing the prediction used in the following iteration.

\section{The Therapists' Interface}

Our platform enables the therapists to manage information about the patients, their sessions and keep an historic of their exercises (Figure 3). Further, it enables them to compare data across sessions or even between patients. In detail, here are the most relevant:

\section{Recording a movement/exercise}

The platform allows the therapist to record a movement for later visualization or comparison by both choosing which points are relevant to the assessment of the movement and placing the sensors (markers) on the patient's body (Figure 4). Then, he/she is able to select them onscreen and match with the desired designation (body part) (Figure 5). This is where we create our internal skeleton representation, a set of restrictions to help the therapist to visualize and compare the movement.

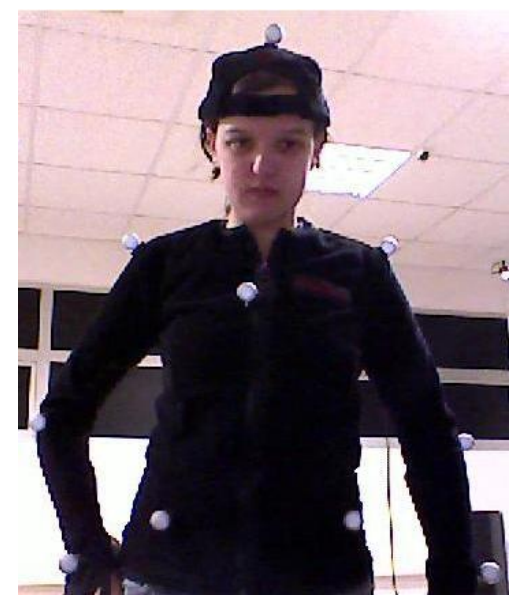

Figure 4 - User equipped with markers

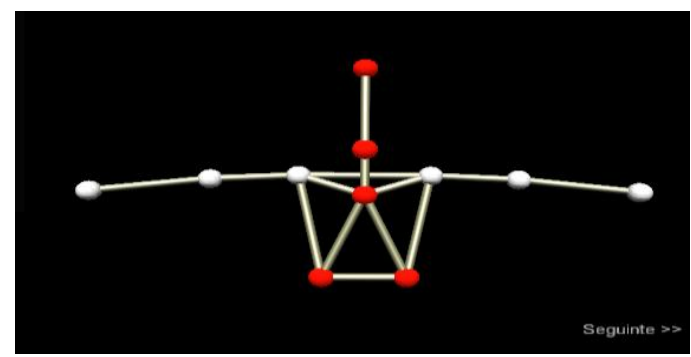

Figure 5 - Selected points in the skeleton

Reproducing a movement/exercise

After recording the movement, the therapists can reproduce it, navigate and look in detail in a threedimensional view (Figure 6). They are able to analyze in detail the points, and observe amplitudes and angles between members. This function behaves like a media player where you can pause, play or even speed up/down a movement.

\section{Comparing movements}

At any time, the therapists can select more than one movement and compare them. To ease the comparison, two different timelines are available (Figure 7). This enables manual control over both movement reproductions. We are currently working on an automatic synchronization to help the therapists finding a good comparison starting point.

\section{SCENARIOS}

The platform's capabilities can be illustrated with the following scenarios:

\section{Capturing and Reproducing a Movement}

Joseph goes every Wednesday morning to therapy. He is tetraplegic and goes to the same clinic since his accident, two years ago. His therapist is called Peter. A few minutes after arriving, Peter began to equip Joseph with sensors. After placing sensors in the shoulders, elbows and wrists, Peter started the application and asked Joseph to put his arms resting on the chair. The therapist selected the patient in the application and introduced the data for the session. He selected the option to record a movement previously added to the database (this means that there is a movement already recorded that features the same tracking points). The movement selected was the one called "Raise Left Arm". The tracked points appeared onscreen already reproducing Joseph's posture. Peter selected each point and attributed its denomination (body part). Upon completion, the skeleton is created (represented by lines

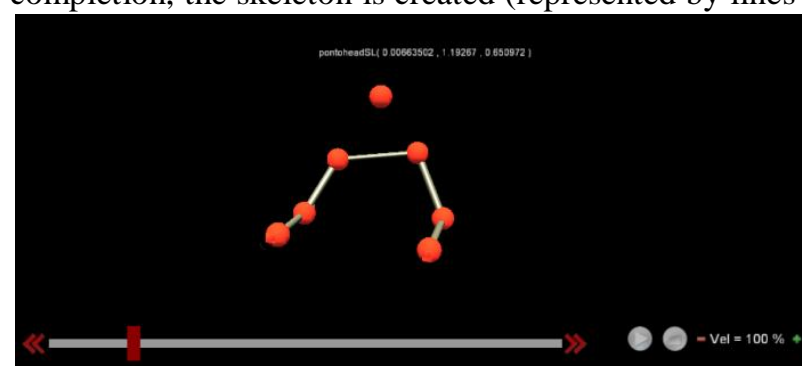

Figure 6 - Movement Reproduction 


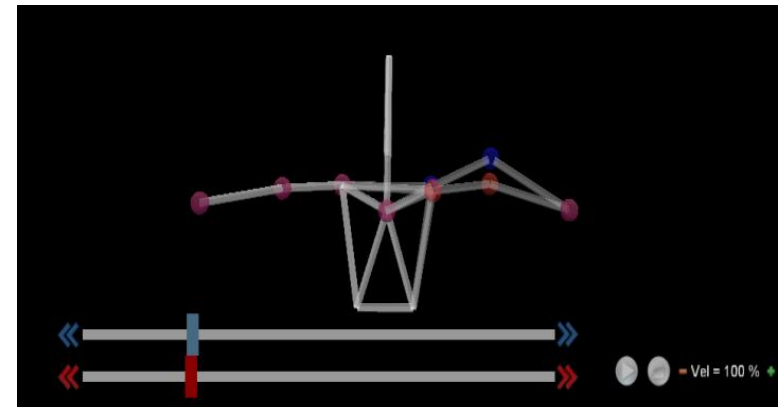

Figure 7 - Comparison between two movements

between points). Peter selected the Record button, asked Joseph to perform the movement, and then saved it.

While observing Joseph, Peter noticed that he was leaning forward while performing the movement. He then selects the previously recorded movement and shows it to Joseph, explaining him what he is doing wrong. By replaying the movement slowly and pausing it as Joseph starts to droop, they can discuss how to avoid it. Joseph could see what he was doing wrong. Peter was also enlightened about the reasons why Joseph was behaving erroneously and feels confident in the exercises to perform in the following sessions to help Joseph improve.

\section{Comparing movements}

After a long working day, Peter intends to conduct an evaluation on the patients' evolution. He began by examining their patients, and check how they are evolving, starting with Andrea. He launched the application, chose the list of sessions and the patient in question. He was then able to see all the movements performed by Andrea in the selected sessions and started to analyze the left arm abilities, selecting the movement saved as "Lift Left Arm". He selected the occurrences of 12/12/2009 and 12/04/2010 and pressed "Compare". He was presented with two bars, one for each run, a three-dimensional skeleton, a play and pause/stop button. He started reproducing the movements by pressing the Play button and felt pleased as he noted that Andrea showed improvements in arm extension; she can now extend the arm at an angle of almost $90^{\circ}$ with the trunk.

\section{PRELIMINARY EVALUATION}

A preliminary analysis with two therapists showed that the application enabled them to complete the tasks they are used to perform a lot quicker as the information is retrieved automatically. Moreover, the therapists acknowledged that the information they have access with the system is much more complete and with higher precision. They enjoyed the system and believe it to be a significant improvement to aid their daily activities.

\section{CONCLUSIONS AND FUTURE WORK}

A task analysis on the rehabilitation procedure and on how therapists observe and evaluate status and evolution of their patients has been presented. The current system is limited concerning the accurate evaluation of the patients' capabilities and evolution patterns. We presented a virtual tracking-based platform that enables the therapists to have immediate and onrecall detailed information about the patients' motions, evolution and overall history. Future work includes evaluating the approach thoroughly and also using the information retrieved from the system to improve the process on the patient's end, e.g., rehabilitation games.

\section{ACKNOWLEDGMENTS}

We would like to thank all the users (both therapists and patients) that participated in our studies. This work was supported by FCT (INESC-ID multiannual funding) through the PIDDAC Program funds. Hugo Nicolau and Tiago Guerreiro were supported by FCT, grants SFRH/BD/46748/2008 and SFRH/BD/28110/2006, respectively.

\section{REFERENCES}

Zhou, H. \& Hu, H.(2004). A Survey - Human

Movement Tracking and Stroke Rehabilitation,

Technical Report.

Arulampalam M., Maskell S., Gordon N., Clapp T. (2002) A Tutorial on Particle Filters for Online Nonlinear/Non-Gaussian Bayesian Tracking, IEEE Transactions on Signal Processing, vol. 50, pp. 174/188.

Welch G., Bishop G. (1995), An Introduction to Kalman Filter, University of North Carolina at Chapel Hill.

Pinho, R. R.; Tavares, J. M. R. S. \& Correia (2005), M. VA Movement Tracking Management Model with Kalman Filtering, Global Optimization Techniques and Mahalanobis Distance, ICCMSE 2005 International Conference on Computational Methods in Science and Engineering. 